\title{
O422 Do the disadvantages of late initiation of HAART persist in patients achieving and maintaining viral load (VL) suppression for a year on HAART? LJ Waters*1, M Fisher ${ }^{2}$, J Anderson ${ }^{3}, \mathrm{C}_{\text {Wood }}{ }^{4}$ and CA Sabin ${ }^{5}$
}

Address: ${ }^{1}$ Chelsea and Westminster Hospital, London, UK, ${ }^{2}$ Brighton and Sussex University Hospital, Brighton, UK, ${ }^{3}$ Homerton University Hospital NHS Foundation Trust, London, UK, ${ }^{4}$ North Middlesex Hospital, London, UK and ${ }^{5}$ Royal Free and UC Medical School, London, UK

* Corresponding author

from Ninth International Congress on Drug Therapy in HIV Infection

Glasgow, UK. 9-13 November 2008

Published: 10 November 2008

Journal of the International AIDS Society 2008, I I (SuppI I):O44 doi:I0.II 86/I758-2652-II-SI-O44

This abstract is available from: http://www.jiasociety.org/content/II/SI/O44

(c) 2008 Waters et al; licensee BioMed Central Ltd.

\section{Purpose of the study}

Starting HAART at low CD4 counts may be associated with impaired CD4 recovery. However, the factors associated with late presentation and delay of HAART may confound any associations with CD4 response. We studied CD4, VL and clinical outcomes over 4 years of HAART in subjects from the UK CHIC Study who maintained VL suppression for the first year on HAART, stratified by preHAART CD4.

\section{Methods}

We identified ARV-naïve adults starting HAART from 1998-2006 (NRTI+NNRTI or PI/r) with pre-HAART CD4 $<350$ cells and VL $>500 \mathrm{cp} / \mathrm{ml}$. Patients were excluded if they had $<1$ year follow-up, they did not suppress VL, they experienced VL rebound in the first year, or they stopped HAART. CD4 rises were compared using Kruskal-Wallis tests in those starting with pre-HAART CD4 of $<100$ (group 1), 101-200 (group 2) or >200 (group 3) cells; VL rebound (>1000 cp/ml) and progression to new AIDS/ death were compared using Kaplan-Meier analysis.

\section{Summary of results}

3,491 of 6,904 ARV-naïve adults starting HAART were eligible. Most were male (74\%), white (56\%), MSM (54\%) with a median age of 36 years. Median follow-up was 3.6 (range 1,9) years after HAART. 1,116 (32.0\%), 1,186 $(34.0 \%)$ and $1,189(34.1 \%)$ were in groups 1,2 and 3 , respectively. 3,029 (87\%) started an NNRTI (mainly EFV) and $13 \%$ a $\mathrm{PI} / \mathrm{r}$-based regimen (mainly LPV). Whilst the median CD4 count was significantly lower in group 1 in all 4 years, the median CD4 increase from pre-HAART was significantly higher in group 1 than in groups $2 / 3$ after 2 , 3 and 4 years (median CD4 increases at year 4 of 345, 320 and 333 cells in groups $1,2 \& 3$ ). The cumulative risk of VL rebound at years 2, 3 and 4 was 6.9, 11.9 and 15.5\%, with no significant difference across groups $(\mathrm{p}=0.10$, logrank test). Group 1 had higher rates of clinical progression than group 3 at 2 years $(1.8 \%$ vs. $0.8 \%)$; this effect persisted over the next 2 years (RHs of 2.10, 1.75 and 2.23 in years 2, 3 and 4 ). Whereas group 2 had a higher rate of clinical progression than group 3 in year 2 after HAART (1.4\% vs. $1.8 \%$ ), this effect weakened (RHs of $1.76,1.21$ and 0.93 in years 2, 3 and 4).

\section{Conclusion}

Individuals starting HAART at CD4 counts $\leq 100$ cells who maintain VL suppression for a year have lower median CD 4 counts by 4 years after HAART. However, CD 4 increases in this group are greater than those in patients starting at higher CD4s, suggesting that any disadvantage may be lost over time if patients can maintain VL suppression. 